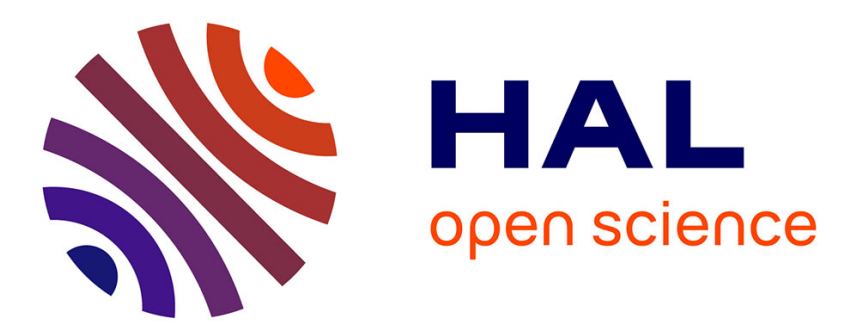

\title{
Multidomain Extension of a Pseudospectral Algorithm for the Direct Simulation of Wall-Confined Rotating Flows
}

\author{
Guillaume Fontaine, Sébastien Poncet, Eric Serre
}

\section{To cite this version:}

Guillaume Fontaine, Sébastien Poncet, Eric Serre. Multidomain Extension of a Pseudospectral Algorithm for the Direct Simulation of Wall-Confined Rotating Flows. M. Azaiez, H. El Fekih, J.S. Hesthaven. Lecture Notes in Computational Science and Engineering, 95, Springer, pp.261 - 271, 2014, 10.1007/978-3-319-01601-6_21 . hal-01098589

\section{HAL Id: hal-01098589 \\ https://hal.science/hal-01098589}

Submitted on 27 Dec 2014

HAL is a multi-disciplinary open access archive for the deposit and dissemination of scientific research documents, whether they are published or not. The documents may come from teaching and research institutions in France or abroad, or from public or private research centers.
L'archive ouverte pluridisciplinaire HAL, est destinée au dépôt et à la diffusion de documents scientifiques de niveau recherche, publiés ou non, émanant des établissements d'enseignement et de recherche français ou étrangers, des laboratoires publics ou privés. 


\title{
Multidomain extension of a pseudospectral algorithm for the direct simulation of wall-confined rotating flows
}

\author{
G. Fontaine, S. Poncet and E. Serre
}

\begin{abstract}
In this work, we improve an existing pseudospectral algorithm, in order to extend its properties to a multidomain patching of a rotating cavity. Viscous rotating flows have been widely studied over the last decades, either on industrial or academic approaches. Nevertheless, the range of Reynolds numbers reached in industrial devices implies very high resolutions of the spatial problem, which are clearly unreachable using a monodomain approach. Hence, we worked on the multidomain extension of the existing divergence-free Navier-Stokes solver with a Schur approach. The particularity of such an approach is that it does not require any subdomain superposition: the value of a variable on the boundary between two adjacent subdomains is treated as a boundary condition of a local Helmholtz solver. This value is computed on a direct way via a so-called continuity influence matrix and the derivative jump of an homogeneous solution computed independently on each subdomain. Such a method is known to have both good scalability and accuracy. It has been validated on two well documented three-dimensional rotating flows.
\end{abstract}

\section{Numerical modelling}

Let's introduce the numerical fundamentals of the present method. A pseudospectral method is used to solve the Navier-Stokes PDE system in an annular cavity, where incompressibility is assured through a projection method.

G. Fontaine, S. Poncet, E. Serre M2P2 Laboratory, Marseilles (France), contact e-mail: guillaume.fontaine@13m.univ-mrs.fr 


\subsection{Pseudospectral methods}

Let $\Omega$ be the inner points of an annular cavity and $\Gamma$ the domain boundary. The spatial approximation is of Chebyshev type in the axial $z$ and radial $r$ directions, of Fourier-Galerkin type in the azimuthal $\theta$ direction. Let $\Psi$ be a variable $(u, v, w, p, \varphi)$, which can be written for any point $(r, \theta, z) \in \Omega \cup \Gamma$ as:

$$
\Psi_{N_{r} N_{\theta} N_{z}}(r, \theta, z)=\sum_{k=-N_{\theta} / 2}^{N_{\theta} / 2} \sum_{n=0}^{N_{r}-1} \sum_{m=0}^{N_{z}-1} \widehat{\Psi}_{n k m} T_{n}(r) T_{m}(z) e^{i k \theta}
$$

where $T_{n}$ and $T_{m}$ are the Chebyshev polynomials of degrees $n$ and $m$ respectively. $N_{r}, N_{z}$ and $N_{\theta}$ are the approximation degrees in the radial, axial and azimuthal directions, respectively. $\widehat{\Psi}_{n k m}$ is given by :

$$
\widehat{\Psi}_{n k m}=\frac{1}{K} \frac{1}{c_{k} c_{m}^{\prime}} \sum_{q=0}^{K-1} \sum_{i=0}^{N} \sum_{j=0}^{M} \frac{1}{c_{i} c_{j}^{\prime}} \Psi\left(r_{i}, \theta_{q}, z_{j}\right) \cos \left(\frac{i n \pi}{N}\right) \cos \left(\frac{i m \pi}{M}\right) e^{-i k \theta_{q}}
$$

$c_{0}=c_{0}^{\prime}=c_{N}=c_{M}^{\prime}=2$ and $c_{k}=c_{m}^{\prime}=1$ and for $n=1, \ldots, N-1$ and $m=1, \ldots, M-1$. This approximation is done using a Gauss-Lobatto point distribution in the radial and axial directions with Fourier-Galerkin points in the azimuthal direction. Thus derivatives can be estimated in the spectral space with a good precision, allowing the use of efficient FFT algorithms. These methods are called pseudospectral because of the non-linear diffusive terms, which must be computed in the physical space.

\subsection{Geometry}

The velocities are made dimensionless according to the Reynolds number $R e=$ $\omega R_{1}^{2} / v$, where $\omega$ is the angular velocity, $R_{1}$ the outer radius of the domain, $v$ the kinematic viscosity. Hence the dimensionless geometry is defined by two parameters: the aspect ratio of the cavity $L=\frac{R_{1}-R_{0}}{2 h}$ and the curvature parameter $R m=\frac{R_{1}+R_{0}}{R_{1}-R_{0}}$, where $R_{1}$ and $R_{0}$ are the outer and inner radii of the cavity, respectively, and $2 h$ its height [3].

\subsection{Projection method}

Let's consider the Navier-Stokes equations in primitive variables :

$$
\begin{array}{r}
\left(\frac{\partial \mathbf{V}}{\partial t}+(\mathbf{V} . \nabla) \mathbf{V}\right)=-\frac{1}{\rho} \nabla p+v \Delta \mathbf{V}+\mathbf{F} \text { in } \Omega \\
\nabla . \mathbf{V}=0 \text { in } \Omega
\end{array}
$$


where $\mathbf{V}$ is the velocity vector with $(u, v, w)$ its components in the cylindrical basis, $p$ the pressure, $\rho$ the density and $\mathbf{F}$ a force term.

\subsubsection{Time discretization}

A semi-implicit second order scheme has been chosen for the time discretization with an implicit retarded Euler scheme of 2nd order for the diffusive terms and an explicit Adams-Bashforth evaluation for the non-linar convective terms.

\subsubsection{Projection scheme}

For incompressible viscous flows, an equation is missing to describe the pressure evolution. We deal with this particularity by using an improved projection method [2] based on the Goda's projection method. It requires 3 steps:

- First step: the computation of a preliminary pressure $\bar{p}^{n+1}$ through this Poisson equation:

$$
\left\{\begin{array}{l}
\nabla^{2} \bar{p}^{n+1}=\nabla \cdot\left[-2 \mathbf{V} \cdot \nabla \mathbf{V}^{n}+\mathbf{V} \cdot \nabla \mathbf{V}^{n-1}+\mathbf{F}^{n+1}\right] \text { in } \Omega \\
\frac{\partial \bar{p}^{n+1}}{\partial n}=\mathbf{n} \cdot\left[\frac{-3 \mathbf{W}^{\mathbf{n}+1}+4 \mathbf{V}^{\mathbf{n}}-\mathbf{V}^{\mathbf{n}-1}}{2 \delta t}-2 \mathbf{V} \cdot \nabla \mathbf{V}^{n}+\mathbf{V} \cdot \nabla \mathbf{V}^{n-1}+\right. \\
\left.v\left(2 \Delta \mathbf{V}^{n}-\Delta \mathbf{V}^{n-1}\right)+\mathbf{F}^{n+1}\right] \text { on } \Gamma
\end{array}\right.
$$

where $\mathbf{W}$ represents the boundary conditions.

- Second step: prediction step. Using the gradient of $\bar{p}^{n+1}$, a preliminary velocity field $\mathbf{V}^{*}$ is computed through 3 Helmholtz solvers:

$$
\left\{\begin{array}{l}
\frac{3 \mathbf{V}^{*}-4 \mathbf{V}^{\mathbf{n}}+\mathbf{V}^{\mathbf{n}-\mathbf{1}}}{2 \delta t}+2 \mathbf{V} \cdot \nabla \mathbf{V}^{n}-\mathbf{V} \cdot \nabla \mathbf{V}^{n-1}=-\nabla \bar{p}^{n+1}+v \Delta \mathbf{V}^{*}+\mathbf{F}^{n+1} \text { in } \Omega \\
\mathbf{V}^{*}=\mathbf{W}^{n+1} \text { on } \Gamma
\end{array}\right.
$$

This velocity field does not a priori satisfy the incompressibility constraint in $\Omega$. The principle of the projection method is namely to project this field on a divergence-free field.

- Third step: pseudo-pressure calculation and correction. An intermediate variable called pseudo-pressure $\varphi$ is computed through a Poisson solver:

$$
\varphi=\frac{2 \delta t}{3}\left(p^{n+1}-\bar{p}^{n+1}\right)
$$

As $\nabla \cdot V^{n+1}=0$, the Poisson problem to solve for $\varphi$ is : 


$$
\left\{\begin{array}{l}
\nabla^{2} \varphi=\nabla \cdot \mathbf{V}^{*} \\
\frac{\partial \varphi}{\partial n}=0
\end{array}\right.
$$

Corrected pressure and velocity fields may be then evaluated at time step $n+1$ :

$$
\left\{\begin{array}{l}
p^{n+1}=\bar{p}^{n+1}+\frac{3}{2 \delta t} \varphi \\
\mathbf{V}^{\mathbf{n}+\mathbf{1}}=\mathbf{V}^{*}-\nabla \varphi
\end{array}\right.
$$

\subsection{Resolution algorithm : complete matricial diagonalisation technique}

For both Poisson and Helmholtz solvers, complete matricial diagonalisation technique is used. For the Poisson solver, this technique exhibits a null-eigenvalue problem, which is treated by a "source term reset" technique ([7]), for indexes in the operators corresponding to the null-eigenvalue index. According to the variable spatial evaluation, each Helmholtz/Poisson solving operation in the physical tridimensional domain is equivalent to $N_{\theta}$ bidimensional Helmholtz/Poisson solving operations in the $(r, z)$ plane, each $k \in\left[\left[1 ; N_{\theta}\right]\right]$ being the azimuthal mode in the flow spectrum [3]. For each mode $k$ and for each variable $\Psi=(u, v, w, p, \varphi)$, the following bidimensional system has then to be solved :

$$
\left\{\begin{array}{c}
\Delta \widehat{\Psi}-\sigma_{k} \widehat{\Psi}=S_{k} \text { in }[-1,1] \times[-1,1] \\
A \Psi=b \text { on } \Gamma
\end{array}\right.
$$

$\widehat{\Psi}$ being the Fourier transform of $\Psi$ in the azimuthal direction. The azimuthal properties of the differentiation matrixes are treated in $\sigma$ so as to allow the $\Delta$ operator to be independant of the azimuthal mode. The azimuthal dependance of what will follow is only linked to $\sigma$ 's one. Hence we will limit the multidomain approach to bidimensional problems, because the extension to tridimensional flows is quite immediate, thanks to spectral properties in the Fourier-Galerkin direction.

\section{Multidomain approach: influence matrix technique}

This technique is a direct Schur multidomain technique used by Raspo [1] for rotating flows using the vorticity-stream function formulation. It requires the patching of the cavity into subdomains without subdomain covering. The values of each variable on the frontier between two subdomains are treated as boundary conditions in 
the Helmholtz local solvers, the particularity of the influence matrix technique being the determination of this condition through a direct matrix computation.

\subsection{Multidomain geometry}

We will limit the discussion to a radial multidomain decomposition (Fig.1), because the curvature terms vary only along this direction. The generalization of this technique to an axial decomposition is quite immediate. We introduce the local aspect ratio of the subdomain $m$ denoted $L^{(m)}$ and its local curvature parameter $R m^{(m)}$ for $m \in[[1 ; M]]$ ( $M$ the number of subdomains), which satisfy :

$$
\begin{array}{r}
\sum_{m=1}^{M} L^{(m)}=L \\
R m^{(m)}-1=R m^{(m+1)}+1
\end{array}
$$

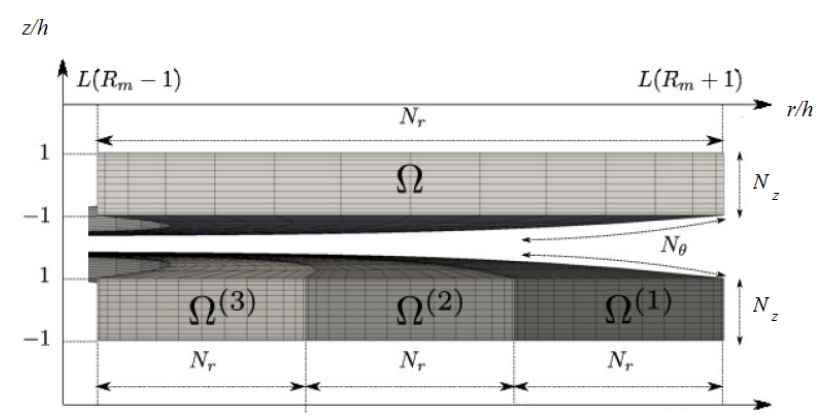

Fig. 1 Example of a multidomain decomposition in the radial direction with 3 subdomains

Local derivation matrixes are deduced from these, in order to have a good approximation for the curvature terms from one subdomain to another and to adapt the local mapping to the one which would be used in a monodomain approach.

\subsection{Multidomain decomposition of the solutions}

Let $\Psi$ be either $(u, v, w, p, \varphi), \Omega^{(m)}$ and $\Omega^{(n)}$ two adjacent subdomains, $\xi$ the border between these two subdomains and $\lambda$ the value of $\Psi$ on $\xi$. For both subdomains, the local problems to be solved may be written as :

$$
\left\{\begin{aligned}
\Delta^{(m)} \Psi^{(m)}-\sigma^{(m)} \Psi^{(m)} & =S^{(m)} \text { in } \Omega^{(m)} \\
A^{(m)} \Psi^{(m)} & =b^{(m)} \text { on } \Gamma^{(m)}
\end{aligned}\right.
$$




$$
\left\{\begin{aligned}
\Delta^{(n)} \Psi^{(n)}-\sigma^{(n)} \Psi^{(n)} & =S^{(n)} \text { in } \Omega^{(n)} \\
A^{(n)} \Psi^{(n)} & =b^{(n)} \text { on } \Gamma^{(n)}
\end{aligned}\right.
$$

The resulting problem is that the boundary conditions to be imposed on the parts of $\Gamma^{(m)}$ and $\Gamma^{(n)}$ corresponding to $\xi$ are unknown. To find $\lambda$, we choose to ensure both $\mathscr{C}^{0}$ and $\mathscr{C}^{1}$ continuities through $\xi . \Psi$ is written as the combination of an homogeneous solution $\widetilde{\Psi}$ and a boundary solution $\bar{\Psi}: \Psi=\widetilde{\Psi}+\bar{\Psi}$.

On $\Omega^{(m)}$ :

$$
\begin{aligned}
& \left\{\begin{array}{r}
\Delta^{(m)} \widetilde{\Psi^{(m)}}-\sigma^{(m)} \widetilde{\Psi^{(m)}}=S^{(m)} \text { in } \Omega^{(m)} \\
A^{(m)} \widetilde{\Psi^{(m)}}=b^{(m)} \text { on } \Gamma^{(m)} \\
\widetilde{\Psi^{(m)}}=0 \text { on } \xi^{(m)}
\end{array}\right. \\
& \left\{\begin{array}{r}
\Delta^{(m)} \overline{\Psi^{(m)}}-\sigma^{(m)} \overline{\Psi^{(m)}}=0 \text { in } \Omega^{(m)} \\
A^{(m)} \overline{\Psi^{(m)}}=0 \text { on } \Gamma^{(m)} \\
\overline{\Psi^{(m)}}=\lambda^{(m)} \text { on } \xi^{(m)}
\end{array}\right.
\end{aligned}
$$

On $\Omega^{(n)}$ :

$$
\begin{gathered}
\left\{\begin{aligned}
\Delta^{(m+1)} \widetilde{\Psi^{(n)}}-\sigma^{(n)} \widetilde{\Psi^{(n)}} & =S^{(n)} \text { in } \Omega^{(n)} \\
A^{(m)} \widetilde{\Psi^{(n)}}=b^{(n)} \text { on } \Gamma^{(m)} & \widetilde{\Psi^{(n)}}=0 \text { on } \xi
\end{aligned}\right. \\
\left\{\begin{aligned}
\Delta^{(n)} \overline{\Psi^{(n)}}-\sigma^{(n)} \overline{\Psi^{(n)}} & =0 \text { in } \Omega^{(m)} \\
A^{(n)} \overline{\Psi^{(n)}} & =0 \text { on } \Gamma^{(n)} \\
\overline{\Psi^{(n)}} & =\lambda \text { on } \xi
\end{aligned}\right.
\end{gathered}
$$

If $\lambda$ is assumed to be known, one can verify easily that (eq. 12)=(eq. 14) $+($ eq. 15) and $($ eq. 13) $=($ eq. 16) + (eq. 17).

\subsection{The influence matrix technique}

Let's consider the boundary solution $\bar{\Psi}$. It can be written as the linear combination of Green's elementary solutions $\mathscr{G}_{k \xi}^{(m)}$, defined for each subdomain $\Omega^{(m)}$ by :

$$
\left\{\begin{array}{l}
\Delta^{(m)} \overline{\mathscr{G}_{k \xi}^{(m)}}-\sigma^{(m)} \overline{\mathscr{G}_{k \xi}^{(m)}}=0 \text { in } \Omega^{(m)} \\
A^{(m)} \overline{\mathscr{G}_{k \xi}^{(m)}}=0 \text { on } \Gamma^{(m)} \\
\overline{\mathscr{G}_{k \xi}^{(m)}}\left(\eta_{l} \in \xi^{(m)}\right)=\delta_{k l} \forall l \in\left[\left[1 ; N_{\xi}\right]\right]
\end{array}\right.
$$

Assuming that $\xi$ is the only boundary (i.e. there are only 2 subdomains $\Omega^{(m)}$ and $\left.\Omega^{(n)}\right)$, the boundary solution should be written as: 


$$
\left\{\begin{array}{c}
\Psi^{(m)}=\widetilde{\Psi^{(m)}}+\sum_{k=1}^{N_{\xi}} \lambda_{k} \mathscr{G}_{k E}^{(m)} \text { in } \Omega^{(m)} \\
\Psi^{(n)}=\widetilde{\Psi^{(n)}}+\sum_{k=1}^{N_{\xi}} \lambda_{k} \mathscr{G}_{k S}^{(n)} \text { in } \Omega^{(n)}
\end{array}\right.
$$

$\widetilde{\Psi^{(m)}} \cup \widetilde{\Psi^{(n)}}$ is obviously continuous through $\xi$, but not $\frac{\partial \widetilde{\Psi^{(m)}}}{\partial r} \cup \frac{\partial \widetilde{\Psi^{(n)}}}{\partial r}$. As the boundary solution is continuous too, $\Psi^{(m)} \cup \Psi^{(n)}$ should be continuous for any value of $\lambda$. The influence matrix technique aims to find $\lambda$ in order to make it $\mathscr{C}^{0}$ and $\mathscr{C}^{1}$ through $\xi$. We denote $\partial_{r}=\frac{\partial}{\partial r}$. This $\mathscr{C}^{1}$-continuity problem on $\xi$ writes:

$$
\widetilde{\partial_{r} \Psi^{(1)}}(\xi)-\partial_{r} \widetilde{\Psi^{(2)}}(\xi)=\sum_{l=1}^{N_{\xi}} \lambda_{l}\left[\partial_{r} \mathscr{G}_{l S}^{(2)}(\xi)-\partial_{r} \mathscr{G}_{l E}^{(1)}(\xi)\right]
$$

This can be written in a matrix form:

$$
\mathscr{D}=\mathscr{M} \lambda
$$

where $\mathscr{D}_{k}$ is the derivative (time-dependent) jump vector and $\mathscr{M}$ the continuity influence matrix of the problem. Note that it depends only on the time-independent Green solutions, so it just has to be computed in pre-processing. This matrix is diagonal-dominant. If there are more than 2 frontiers in the domain, the influence matrix is built by blocks. The block dimension is then $N_{\text {front }}$, the number of frontiers. Each diagonal block is a Green derivative jump vector along each frontier. Some non-diagonal blocks appear, resulting locally of the influence of 2 frontiers on one another through a single subdomain, as shown on Figure (2).

If $\mathscr{M}$ is inversible, we can find $\lambda$ as:

$$
\lambda=\mathscr{M}^{-1} \mathscr{D}
$$

This is achieved using LAPACK subroutines. This vector is then used as a boundary condition on $\xi$ in the local Helmholtz solvers to get a $\mathscr{C}^{0}$ and $\mathscr{C}^{1} \Psi^{(m)} \cup \Psi^{(n)}$ solution.

\subsection{Singularity of the Poisson-problem}

The Neuman-Poisson problem has an infinity of solutions defined up to an additive constant. As Dirichlet boundary conditions are implemented on the frontiers, this problem no longer exists locally. Nevertheless, it is transposed to the influence matrix of the Poisson-problem of the $k=0$ Fourier mode, which has a null-eigenvalue. It is treated by a diagonalisation technique of this modal matrix. The derivative jump is expressed in the diagonalisation basis and its $i_{0}$-th component is set to zero, if $i_{0}$ is the null eigenvalue index, as proposed by Abide and Viazzo [7]. 
$\mathcal{F}^{i}=$ derivative jump between any elem. solution of $\xi_{i}$ from $\Omega_{i}$ and any elem. solution of $\xi_{i}$ from $\Omega_{i+1}$

$\mathcal{U}^{i}=$ influence of the top ("up") of $\Omega_{i}$ on $\xi_{i}$

$\mathcal{D}^{i+1}=$ influence of the top ("Dwn") of $\Omega_{i+1}$ on $\xi_{i}$

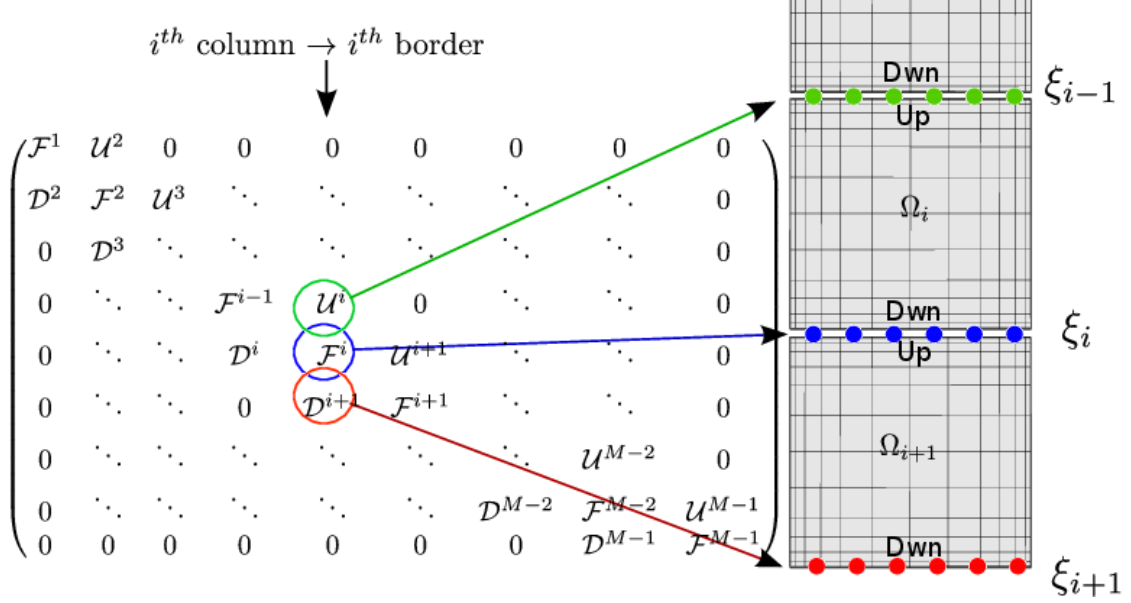

Fig. 2 Block definition of the influence matrix for an 1-D multidomain decomposition

\section{Spatial accuracy}

Let's consider a domain $\Omega$ subdivided in $M$ subdomains with $N_{r}$ grid points in each subdomain. The total number of points may vary either with $M$ or $N_{r}$. For $(r, z, \theta) \in[-1,1] \times[-1,1] \times[0,2 \pi]$, let's consider the divergence free analytical steady solution introduced by Raspo et al. [2]:

$$
\left\{\begin{array}{r}
u_{\text {ana }}(r, z, \theta)=\frac{1}{2 \pi} \sin (\pi r)^{2} \sin (2 \pi z) \cos (\theta) \\
v_{\text {ana }}(r, z, \theta)=-\frac{1}{2 \pi} \sin (\pi r)^{2} \sin (2 \pi z) \sin (\theta) \\
w_{\text {ana }}(r, z, \theta)=\frac{1}{2 \pi L} \sin (\pi z)^{2} \sin (2 \pi r) \cos (\theta) \\
p_{\text {ana }}(r, z, \theta)=[\cos (\pi z)+\cos (\pi r)] \cos (\theta)
\end{array}\right.
$$

Figures 3 and 4 show the decrease of the quadratic truncature error $L^{2}$ when one increases $N_{r}$ and $M$ respectively. In the first case, the spectral convergence is obtained. As we impose only a $\mathscr{C}^{1}$ continuity through the frontier, the spatial accuracy increases indeed faster with $N_{r}$ than with $M$, because in its last case, it multiplies the number of interfaces and so the number of $\mathscr{C}^{d}$ discontinuities $(d \geq 2)$. 
Fig. 3 Spatial accuracy evolution with the total number of points $\left(N_{r} N_{z} N_{\theta} M\right)$ when $N_{r}$ varies : spectral convergence

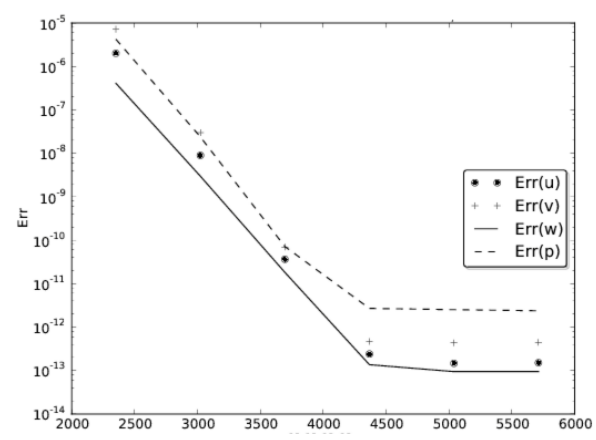

Fig. 4 Spatial accuracy evolution with the total number of points $\left(N_{r} N_{z} N_{\theta} M\right)$ when $M$ varies : no spectral convergence

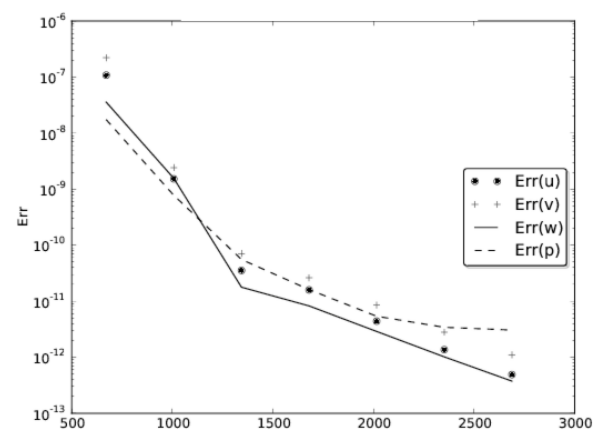

\section{Physical results}

We consider two multidomain rotating flow configurations: a high aspect ratio Taylor-Couette system and an interdisk rotor-stator flow.

\subsection{Axial decomposition : Taylor-Couette flow}

The numerical parameters are fixed to $M=7,\left(N_{r}, N_{\theta}, N_{z}\right)=(35,24,85)$ (in each subdomain) with a time step set to $\delta t=10^{-3}$. We consider a Taylor-Couette cavity with rotating terminal disks characterized by $L=0.025$ and $R m=12.33$. We introduce the parameter $\varepsilon=T a / T a_{c}$, where $T a_{c}$ is the critical Taylor number at which "Taylor rolls" appear. For $\varepsilon=1.36$, the flow becomes tridimensional (Wavy Vortex Flow, Fig.6) with a dominant Fourier mode $k=3$, which is in perfect agreement with the previous DNS results of Serre et al. [6]. 
Fig. 5 Iso-value $v=0.5$ for a Wavy Vortex Flow at $\varepsilon=1.36$

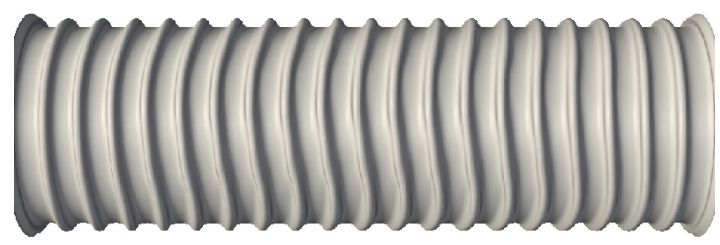

\subsection{Radial decomposition: interdisk rotor-stator flow}

We consider the rotor-stator interdisk cavity considered by Poncet et al. [5] for which $L=6.26$ and $R m=1.8$. The domain is decomposed into 4 subdomains with $\left(N_{r}, N_{\theta}, N_{z}\right)=(45,48,45)$ in each subdomain and a time step equal to $\delta t=10^{-4}$. The transition to tridimensional flow appears at $R e=25000$ with the appearance of 17 spiral arms, whose characteristics fully agree with the ones obtained by [5].

Fig. 6 Iso-value $w=0.005$ for a $3 \mathrm{D}$ rotor-stator flow at $R e=25000: 17$ spiral arm structures

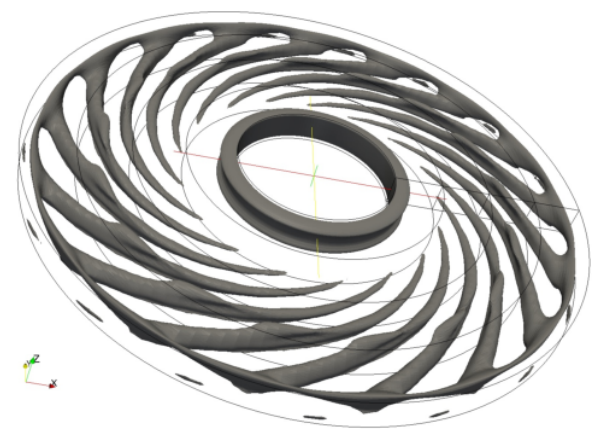

\section{References}

1. Raspo, I.: A direct spectral domain decomposition method for the computation of rotating flows in a T-shape geometry. Comput. Fluids 32, 431-456 (2003)

2. Raspo, I., Hugues, S., Randriamampianina, A., Bontoux, P.: A spectral projection method for the simulation of complex three-dimensional rotating flows. Comput. Fluids 31, 745-767 (2002)

3. Serre, E.: Instabilité de couche-limite dans les écoulements confinés en rotation. Simulation numérique directe par une méthode spectrale de comportements complexes. Phd thesis, Univ. Aix-Marseille II (2000)

4. Peyret, R.: Spectral methods for incompressible viscous flow. Springer, New-York (2002)

5. Poncet, S., Serre, E., Le Gal, P.: Revisiting the two first instabilities of the flow in an annular rotor-stator cavity. Phys. Fluids 21, 064106 (2009)

6. Serre, E., Sprague, M., Lueptow, M.: Stability of Taylor-Couette flow with radial throughflow. Phys. Fluids 20, 034106 (2008)

7. Abide, S., Viazzo S.: A 2D compact fourth-order projection decomposition method. J. Comp. Phys. 206, 252276 (2005) 